\title{
Delirium secundario a abstinencia de nicotina: un problema poco conocido en la Unidad de Cuidados Intensivos
}

\author{
Delirium from nicotine withdrawal \\ in a post-operative adult patient
}

$\mathrm{S}_{\mathrm{r}}^{\mathrm{r}}$ Editor: El delirium, de acuerdo al DSM-IV, es n trastorno agudo de la conciencia y funciones cognitivas ${ }^{1}$, y constituye un problema frecuente en pacientes hospitalizados, con una prevalencia de $6 \%$ a $56 \% 2$. Sus características clínicas incluyen confusión fluctuante, desorientación, dificultades en focalizar la atención, agitación motora y alucinaciones ${ }^{1}$. Puede acompañarse de manifestaciones autonómicas como taquicardia, fiebre e hipertensión.

Dada su alta incidencia y asociación con morbilidad y mortalidad es importante reconocerlo y tratarlo prontamente. El diagnóstico diferencial es amplio y la etiología muchas veces es multifactorial, siendo las causas más frecuentes alteraciones metabólicas, infección, intoxicación o abstinencia de fármacos. La abstinencia a nicotina no es considerada frecuente como causa de delirium: existen menos de 10 comunicaciones en la literatu$\mathrm{ra}^{3,4}$. Damos a conocer a un paciente hospitalizado en la Unidad de Cuidados Intensivos con delirium e historia de consumo importante de tabaco, quien mostró una rápida e importante mejoría de los síntomas luego del tratamiento con un parche transdérmico de $30 \mathrm{mg}$ de nicotina.

Un hombre de 53 años, empresario, fumador de 30 cigarrillos al día por 20 años, y con dislipidemia controlada con régimen, consultó a un cardiólogo por fatigabilidad, detectándosele un soplo aórtico. El estudio ecocardiográfico transtorácico, TAC de tórax y coronariografía confirmaron una valvulopatía aórtica y una anuloaortoectasia marcada de la aorta ascendente. Se realizó un reemplazo de la raíz aórtica y de la aorta ascendente con un tubo valvulado; la cirugía se desarrolló sin incidentes. Antes de $24 \mathrm{~h}$ del postoperatorio presentó agitación

Correspondencia a: Dr. Marcelo Miranda, Antonio Varas 175 of 302. Santiago de Chile. E-mail: marcelomiranda@terra.cl psicomotora con desorientación, fallas atencionales, insomnio y ocasionalmente alucinaciones visuales. Este cuadro confusional evolucionó sin cambios por más de una semana, sin respuesta a la infusión continua de haloperidol y luego olanzapina $5 \mathrm{mg}$ por día, sugeridos por psiquiatra interconsultor, más lorazepam $2 \mathrm{mg}, 2$ a 3 dosis diarias. Recibió desde el $2^{\underline{0}}$ día del postoperatorio un parche de $20 \mathrm{mg}$ de nicotina. Tanto el electroencefalograma estándar como un scanner de cerebro no mostraron alteraciones. En el examen neurológico destacaba falla atencional fluctuante, con incapacidad de retener 3 objetos al cabo de $5 \mathrm{~min}$, desorientación témporo-espacial, sin fallas formales del lenguaje y sin anormalidades focales.

Al noveno día se decidió aumentar la concentración del parche de nicotina a $30 \mathrm{mg}$ diarios con lo que en menos de $4 \mathrm{~h}$ estuvo lúcido, orientado, sin agitación ni insomnio. No recurrieron los episodios confusionales, siendo dado de alta a los pocos días.

En el diagnóstico diferencial en este paciente también se planteó una encefalopatía asociada a la circulación extracorpórea. Este trastorno cede habitualmente en menos de una semana, no se asocia con alucinaciones llamativas y no hubo eventos de hipotensión relevantes en la cirugía que hubieran favorecido su aparición. Por otro lado, la rápida respuesta a una dosis mayor de nicotina, que ya ha sido observada por otros autores, cuando hay falla a una dosis inicial baja ${ }^{3}$, apoya claramente el diagnóstico de delirium secundario a abstinencia a nicotina y ha sido sugerido como test confirmatorio ${ }^{3,4}$.

La nicotina es el principal alcaloide del tabaco, responsable de su capacidad adictiva. Se absorbe rápidamente y llega al cerebro en 10 a $12 \mathrm{~s}$, provocando liberación de diversos neurotransmisores (dopamina, serotonina, noradrenalina y acetilcolina); el estímulo dopaminérgico del sistema 
de recompensa mediado por el núcleo accumbens explica la adicción que provoca la nicotina ${ }^{5}$. Este núcleo límbico es una vía común para las sensaciones placenteras y lugar de acción de la mayoría de las drogas adictivas ${ }^{6}$.

En humanos, la inyección de nicotina resulta en un aumento de la actividad neuronal en regiones límbicas y corticales, tales como amígdala, núcleo accumbens, el cíngulo y lóbulos frontales. Todo esto explica los efectos potenciadores conductuales inducidos por la nicotina en el sistema nervioso central: placer, alivio de ansiedad, aumento de capacidad de realizar labores, aumento de la memoria, modulación del ánimo y relajación muscular ${ }^{6}$. Al bajar los niveles de nicotina los fumadores dependientes comienzan a presentar síntomas de abstinencia que incluyen ira, irritabilidad, ansiedad, insomnio, dificultades en la concentración, somnolencia, fatiga, hambre, aumento de peso, impaciencia, inquietud, ánimo depresivo y más raramente delirium ${ }^{6}$. Estos síntomas comienzan en las primeras $24 \mathrm{~h}$, alcanzan un máximo en 1 a 2 semanas, y generalmente se disipan al cabo de 1 mes.

\section{REFERENCIAS}

1. American Psychiatric Association. Diagnostic and Statistical manual of mental disorders. $4^{\text {th }}$ edition Washington. American Psychiatric Press 1994.

2. Inouye SK. Delirium in hospitalized older patients. Clin Geriatr Med 1998; 14: 745-64.

3. Mayer S, Chong J, Ridgway E, Min K, Comnichau C, BERNARdinI G. Delirium from nicotine withdrawal in neuro-ICU patients. Neurology 2001; 57: 551-3.
Esta comunicación enfatiza que debe considerarse a la abstinencia de nicotina como causa de delirium en pacientes hospitalizados con antecedente de tabaquismo y que el simple uso de un parche de nicotina de $20 \mathrm{mg}$, y si no hay respuesta insistir con $30 \mathrm{mg}$ diarios, puede ofrecer una respuesta clínica muy favorable y además constituye una herramienta diagnóstica para evitar mayor estudio y prolongación de la estadía hospitalaria.

La OMS estima que en el mundo existen 1.100 millones de personas con tabaquismo. Considerando que $22,8 \%$ de los adultos son fumadores y $50 \%$ a $80 \%$ de ellos presentan dependencia a la nicotina ${ }^{5}$, es probable que estemos subdiagnosticando esta entidad.

\section{Marcelo Miranda C. \\ Andrea Slachevsky Ch. Patricio Venegas $P$.}

Unidad de Neurología y Cardiología de Clínica Las Condes, Santiago de Chile.

4. Kiein M, Payasuan S, Gómez J, Prigioni A. Acute confusional syndrome due to acute nicotine withdrawal. Medicina 2002; 62: 335-6.

5. ZhiKowski S, Swan G, McClure J. Cigarette smoking and nicotine dependence. Med Clin N Am 2004; 88: 1453-65.

6. Talwar A, Jain M, Vijayan V. Pharmacotherapy of tobacco dependence. Med Clin N Am 2004; 88: 1517-34.

\section{FE DE ERRATUM}

En el Artículo de Investigación "Curva nacional de distribución de peso al nacer según edad gestacional. Chile, 1993 a 2000", de los autores R González, R Gómez, R Castro, J Kae, P Merino, A Etchegaray et al, Rev Méd Chile 2004; 132: 1155-1165, en Introducción, último párrafo, dice:

“... la mortalidad perinatal era de $8,6 \%$ y $8,9 \%$ por 1.000 recién nacidos vivos...".

Debe decir:

“... era de 8,6 y 8,9 por 1.000 recién nacidos vivos...". 